\title{
HyKim - Development of a robot bear: Bringing the strength and robustness of a bear's biomimetic features to a robot.
}

\author{
Peter Turner \\ School of Electrical Engineering \\ and Computer Science \\ University of Newcastle \\ Callaghan 2308, NSW, Australia
}

Peter.Turner@newcastle.edu.au

\begin{abstract}
There are many commercially available robots that display biomimetic influences. Sony's Aibo and DasaRobot's Genibo are two examples where robot dog designs have drawn from nature. Aldebaran's Nao and Hanson Robotics' Zeno are examples of humanoids robots that have drawn influences from the human body. This paper presents the design of an autonomous 21 Degree of Freedom (DOF) robot bear, named HyKim and discusses the relevant biomimetic influences.

After discussing the motivation for creating a robot bear, the biomimetic principles that were applied to the mechanical design, to ensure the resulting robot was 'bear-like', are presented. The design of the computer and electronic architecture was based on four essential design criteria - open architecture, performance, modularity and reliability. How these criteria were met is then presented, followed by a discussion on future research projects that will be based on or include HyKim. Finally a conclusion summarising the design is presented.
\end{abstract}

Index Terms - Robotics, Biped, Quadruped, Biomimetic, Bear

\section{INTRODUCTION}

When Sony announced it was ceasing production of its popular quadrupedal robot dog, Aibo [1] the future of RoboCup's [2] 4-Legged League (4LL) [3] was put into doubt. The 4LL was one of the most popular RoboCup soccer events as the Aibo's were visually appealing and could play a fast paced and hence exciting game. Exhibiting stable quadrupedal motion allowed the competing programmers to develop complex game strategies with some implementing complex team work, such as passing between team members. The 4LL was also a popular league amongst the competitors, as there was a standard hardware platform the only competitive edge that could be achieved was by developing superior algorithms.

The NUBot's [4] from the University of Newcastle have been a very successful team in the $4 \mathrm{LL}$, having been world champions in 2006 and finishing in the top three teams at all RoboCup's in which they had participated, 2002 to 2007. The NUBot's team leaders formed a project team to investigate suitable replacements for the Sony Aibo, so that the 4LL could continue. In 2006 a prototype quadruped robot $[5,6]$ that may replace the Sony Aibo was presented. At this time

\author{
Michael Dickinson \\ School of Design, Communication \\ and Information. Technology \\ University of Newcastle \\ Callaghan 2308, NSW, Australia
}

Michael.Dickinson@newcastle.edu.au



Fig. 1 Image showing HyKim in a quadrupedal and bipedal stance.

the concept of a quadruped robot that could stand as a biped, for better viewing perspective, was discussed and initial work on the feasibility of achieving this commenced. The concept of a robot bear was introduced in $[5,6]$, this paper will expand on these concepts and present our completed design.

HyKim [7], a 21 Degree Of Freedom (DOF) robot bear, is the result of this project and is shown in Fig. 1. Biomimetic principles were adopted to create this robotic bear. Bear anatomy $[8,9,10,11]$ and bear movements [12] were closely studied so that the resulting robot could be as 'bear-like' as possible. To achieve this a DOF was required at the hip and the shoulders, this resulted in a segmented body. Ref [13] discusses how the challenge of making the robot bear aesthetic and appealing was achieved.

At the outset of the project, a number of essential design criteria were established, these were:

1) Open Architecture: all software provided must be open-source whilst block diagrams and schematics must be made available to the researchers using this robotic platform.

2) Performance: the resulting robot must have high performance servo motors, a powerful processing system and a range of sensors that covers all conceivable requirements. 
3) Modularity: the resulting robot must be modular, both mechanically and electronically, to ensure future upgrades and maintenance could be completed quickly and easily.

4) Reliability: the resulting robot must be robust and durable enough to withstand repeated usage.

HyKim's development is now complete. HyKim can now be used as development platform for future research on optimal and realistic quadrupedal and bipedal gaits.

\section{MECHANICAL ARCHITECTURE}

\section{A. Biomimetics}

Initially our development was based around a Terrier style quadruped robot $[5,6]$. One of the main problems that had to be overcome in this design was creating a physically sleek body that was 'dog-like' whilst incorporating bulky electronic modules. Two of the defining characteristics of a dog are its thin legs and the tapering of its body from the chest to the hip region. Achieving this body shape with readily available electronic modules (servo motors and battery packs) was extremely difficult.

A bear however is a large, stocky animal. As a robot bear would also have this excess bulk, designing a robot bear would allow greater freedom in the selection of processing systems, servo motors and battery packs. Another attraction in selecting a bear as the basis for a robot was the ability of the bear to achieve quadrupedal and limited bipedal motion. In fact a bears walk is plantigrade, heals down with flat feet, the same as humans.

A Polar Bear has an elongated snout [10] in comparison to other bear species. Basing HyKim's design around a Polar Bear allowed us to utilise this fact to house some of the electronic systems in bear's head. After extensive research into bear anatomy $[8,9,10,11]$ and motions [12] a scaled mechanical design based around a polar bears skeleton was created, this is shown in Fig. 2. This initial structure was the basis of aesthetic development that led to the robot seen in Fig 3. Aesthetic development is addressed in [13].

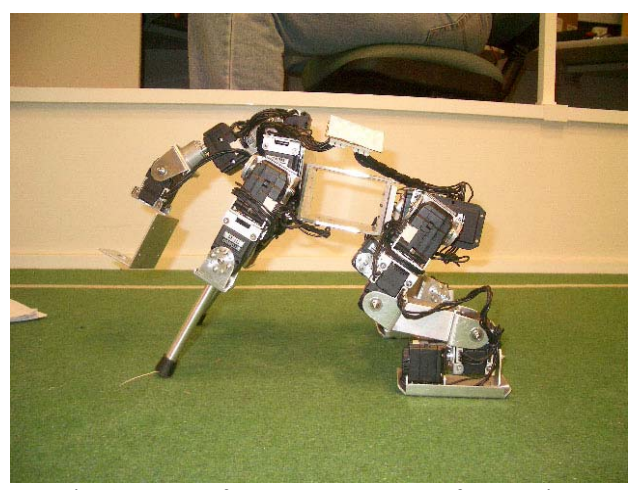

Fig. 2 Image of prototype structure for HyKim.

\section{B. Simplicity}

Our mechanical design required that the structure of HyKim be as simple as possible, i.e. the number of custom designed body pieces be kept to a minimum. This was one consideration when selecting the servo modules. Selecting
Robotis's RX range of Dynamixels not only gave us a high performance servo module but allowed us to utilise the complementary precision aluminum hinge and mounting brackets. For HyKim to be a true kit-style robot, an easy to assemble structure was essential.

TABLE I

HYKIM's PHYSICAL CHARACTERISTICS

\begin{tabular}{|c|c|c|c|}
\hline \multicolumn{2}{|r|}{ Body } & \multicolumn{2}{|c|}{ Degrees of Freedom (DOF): 21} \\
\hline Height & $0.59 \mathrm{~m}$ & Head & $2 \mathrm{DOF}$ \\
\hline Weight & $5.0 \mathrm{~kg}$ & Neck & $1 \mathrm{DOF}$ \\
\hline & & Shoulder & $1 \mathrm{DOF}$ \\
\hline & Battery & Front Legs & 3 DOF $\times 2$ \\
\hline Type & Lithium Polymer & Hip & $1 \mathrm{DOF}$ \\
\hline Voltage & $14.4 \mathrm{~V}$ & Back Legs & 3 DOF $\times 2$ \\
\hline Capacity & $2400 \mathrm{mAh}$ & Ankle & 2 DOF $\times 2$ \\
\hline
\end{tabular}

\section{Kinematics}

Table I details physical characteristics of HyKim. HyKim stands $0.59 \mathrm{~m}$ high and weighs $5.0 \mathrm{~kg}$. HyKim has 21 Degrees of Freedom (DOF) which allow it to display quadrupedal and some bipedal movements.

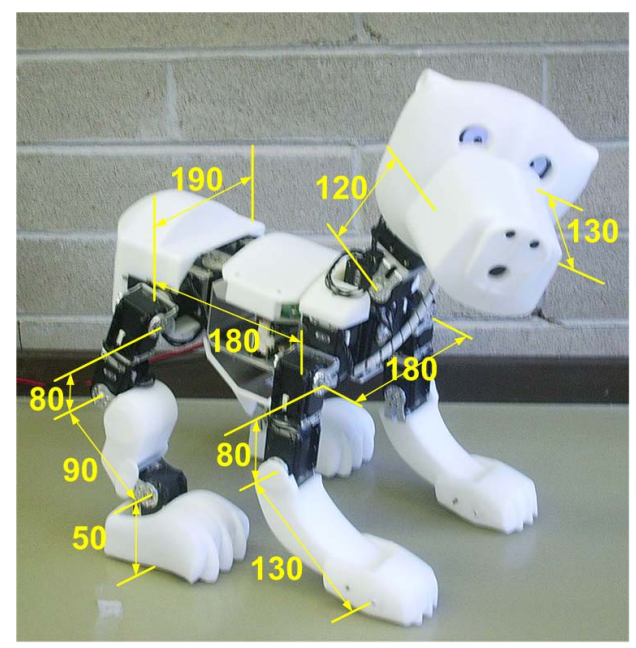

Fig. 3 HyKim at a later development stage with key biomimetic dimensions. N.B. Dimensions are from centre of DOF.

HyKim's dimensions are listed in Table II. As the data shows, as a quadruped HyKim is approximately one and a half times as big as a Sony Aibo [1] whilst as a biped HyKim is of an equivalent size and weight to the Aldebaran Nao [14].

TABLE II

HYKIM's DIMENSIONS

\begin{tabular}{|c|l|c|c|c|}
\cline { 3 - 4 } \multicolumn{2}{c}{} & \multicolumn{2}{c|}{ Measurement $(\mathrm{mm})$} & \multicolumn{1}{c}{} \\
\hline$l_{1}$ & Description & HyKim & Sony Aibo & Size Ratio \\
\hline$l_{2}$ & Neck & 120 & 100 & 1.20 \\
\hline$l_{3}$ & Head Length & 130 & 130 & 1.00 \\
\hline$l_{4}$ & Upper Front Leg & 80 & 70 & 1.14 \\
\hline$l_{5}$ & Lower Front Leg & 130 & 75 & 1.73 \\
\hline$l_{6}$ & TOTAL Front Leg & 210 & 145 & 1.45 \\
\hline$l_{7}$ & Front Width & 180 & 130 & 1.38 \\
\hline$l_{8}$ & Upper Back Leg & 80 & 70 & 1.14 \\
\hline$l_{9}$ & Lower Bag Leg & 90 & 75 & 1.20 \\
\hline$l_{10}$ & Ankle Back Leg & 60 & N/A & N/A \\
\hline$l_{11}$ & TOTAL Back Leg & 230 & 145 & 1.58 \\
\hline$l_{12}$ & Back Width & 190 & 130 & 1.45 \\
\hline$l_{13}$ & Back Length & 180 & 130 & 1.38 \\
\hline
\end{tabular}


HyKim differs significantly from other commercially available quadrupeds, Sony Aibo [1] and DasaRobot Genibo [15], in the following ways:

1) Structure: HyKim's internal structure is made entirely from Aluminum, giving a strong whilst light weight frame.

2) Head and Neck: HyKim has a 3 DOF head and neck design. This coupled with the fact the head has it's own microcontroller with a 6-axis Inertial Measurement Unit (IMU) allows for an independent head stability control algorithm to be implemented, reducing the head bounce whilst walking thus reducing the image processing requirements of the main processing module, HyInt.

3) Shoulder: HyKim has a DOF that allows its shoulders to rotate around the axis of the backbone.

4) Hip: HyKim has a DOF at the lower part of its back that allows the upper torso to move in a side-to-side motion.

5) Legs: HyKim has different front and rear legs, in both configuration and length, as well as a controllable ankle DOF in each of the rear legs.

The shoulder and hip DOF resulted in a segmented body. The three segments being a rear leg section, a mid-section where the main electronic systems could be housed and an upper body section. This segmentation allows for realistic bear gaits to be implemented but does introduce significant complexity in the design of the body covering as discussed in [13]. Fig. 4 shows how HyKim is able to emulate a bears lumbering and plodding style gait due to the additional DOF at the hip and shoulder.

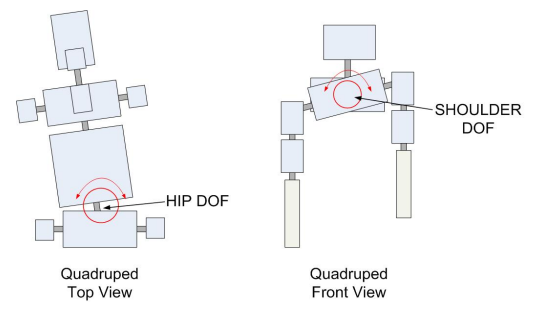

Fig. 4 Additional DOF in Hip and Shoulder of HyKim allow for advanced Quadruped gaits

Fig. 5 shows how HyKim's hip DOF can be used to compensate for a changing Center of Gravity (COG) whilst performing bipedal motions. The hip DOF is not seen in commercially available humanoids used for research purposes, Aldebaran Nao [14] and Robotis's URIA [16], or humanoids with strong biomimetic influences, Hanson Robotics' Zeno [17].

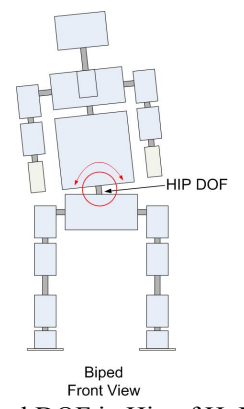

Fig. 5 Additional DOF in Hip of HyKim allows for COG compensation for Biped gaits

\section{Modularity}

One of the key design goals of HyKim was for a modular design, both mechanically and the electronically. This has been achieved mechanically by the:

1) segmented body design,

2) head and neck being a modular design, and

3) the legs also being modular.

This modularity allows for easy upgrades and maintenance.

HyKim was originally designed to be a soccer playing robot, similar to the Sony Aibo. One current characteristic of quadruped robot soccer is that all robots have the same physical characteristics, e.g. RoboCup 4 Legged League (4LL). This is in direct contrast to human soccer games where all the competitors have different body shapes. Using our modular design it is possible to change each robots characteristics, e.g. longer or shorter legs, so that players are optimised for certain positions.

\section{E. Durability}

Another requirement for HyKim was that it be extremely durable. From our experience designing quadruped and biped robots the only way to guarantee this durability is to use servo modules that use metal gears. Robotis's RX range of Dynamixels also met this criterion by providing full metal gears in the Dynamixels used, RX-28 and RX-64.

Robotis's Dynamixels have three other desirable characteristics that improve robot durability, these are:

1) temperature monitoring: a maximum operational temperature can be set in each module, by default this is $80^{\circ} \mathrm{C}$.

2) positioning boundary: a maximum and minimum angular position can be defined in each Dynamixel.

3) torque limiting: a maximum torque for each Dynamixel can be defined.

The Dynamixels can be programmed to either simply alarm or shut down when any of these three conditions occur.

\section{COMPUTER \& ELECTRONICS ARCHITECTURE}

\section{A Computer Architecture}

Fig. 6 shows the block diagram of HyKim's processing system. In all there are twenty five microcontrollers making this is a highly distributed architecture consisting of four major components:

1) HyInt: main processing system,

2) HySense: a data acquisition module located in the centre of the body that communicates by RS485 to HyInt,

3) HySense Lite: a data acquisition module located in the head that communicates by RS485 to HyInt, and

4) Dynamixel RX modules: $4 \mathrm{RX}-64$ and $17 \mathrm{RX}-28$ servo modules that communicate to HyInt on two separate $1 \mathrm{Mbps}$ RS485 networks.

HyInt is HyKim's central processing system which consists of three boards. The main board is a computer module (CM) from Compulab, the CM-iGLX. This CM is based around a 500MHz AMD Geode and has $256 \mathrm{Mb}$ DDR SDRAM, 512Mb FLASH Disk and on-board 802.11g. 


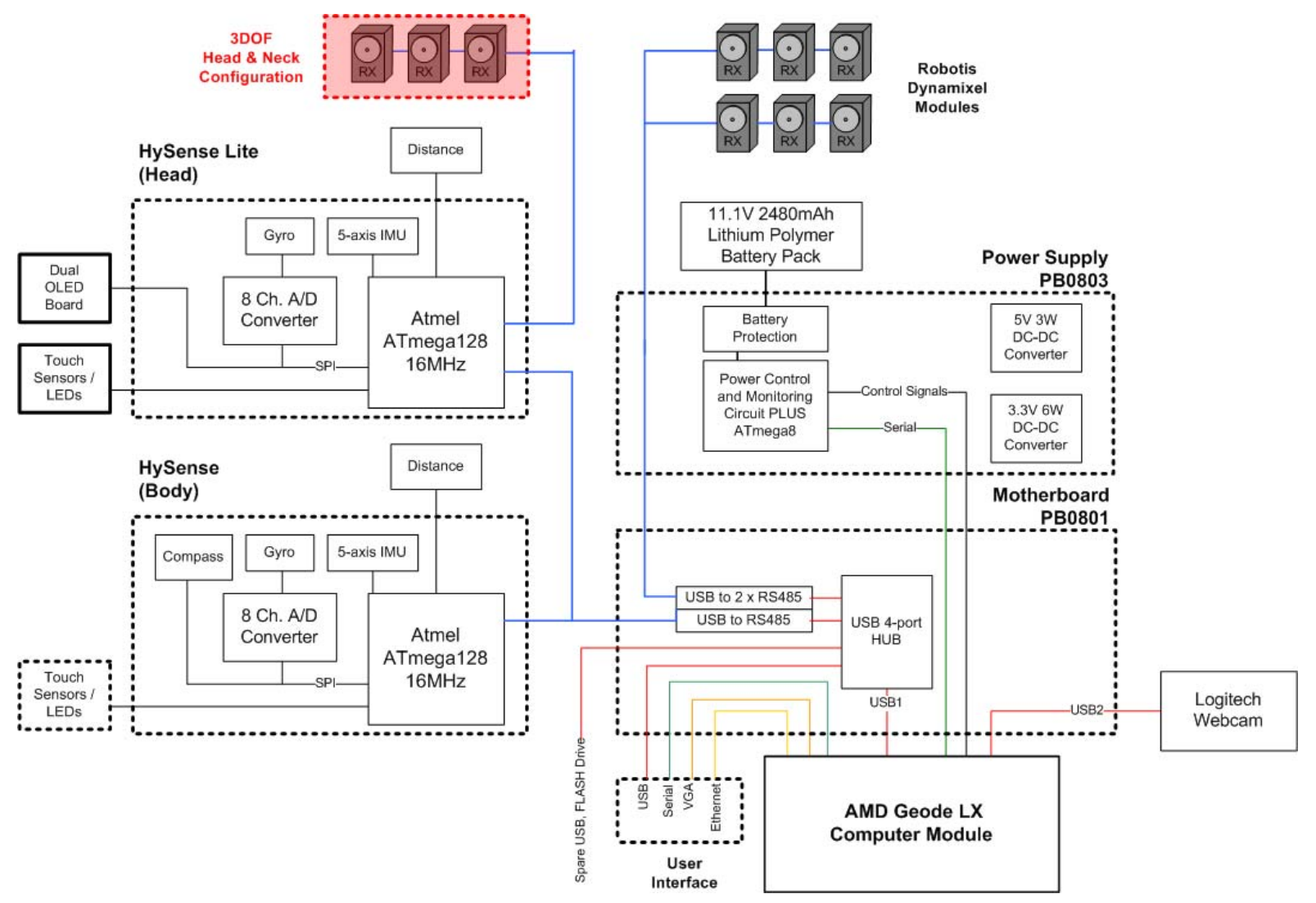

Fig. 6 Block Diagram of HyKim's processing systems.

Our original design for HyInt consisted of dual Intel PXA-255 400MHz microcontrollers which were chosen for their low power consumption, $0.2 \sim 3 \mathrm{~W}$. A dual Intel XSCALE PXA-255A system would provide a total of 500MIPS. The AMD Geode LXMCU provides 990MIPS plus $270 \mathrm{Mflops}$ for $3 \sim 5 \mathrm{~W}$ of power consumption. The decision to switch from a dual Intel XSCALE PXA-255 system to a single AMD Geode LXMCU system gave a better Power vs MIPS and Mflops ratio.

The two additional boards that make up HyInt provide an Atmel ATMega8 for power monitoring, circuitry for three 1Mbps RS485 networks and a spare USB port for a FLASH drive. A fourth board can be connected to HyInt which gives access to the CM-iGLX's Ethernet port, VGA port, USB keyboard and COM port.

HySense and HySense Lite are based around Atmel's ATMega128. Both these modules perform the role of data acquisition modules with the ability to do pre-processing of acquired data before it is transmitted to HyInt by the high speed RS485 network. As the name suggests HySense Lite is a reduced function version of HySense but for programming convenience shares the same General Purpose IO (GPIO) allocation.

Both HySense and HySense Lite have been mounted in central locations near their sensors, this was done primarily to shorten the cable length and thus reduce induced noise.
HySense is physically located on HyKim's middle body segment while HySense Lite is located in HyKim's head.

The fourth major component of HyKim is the network of Dynamixel RX modules. Two 1Mbps RS485 networks are used to communicate with the Dynamixels distributed around HyKim's body. One of the RS485 networks is used for the rear of the robot, rear legs and hip, while the other is used for the front of the robot, front legs, shoulder, head and neck. The Dynamixel RX modules are the core design component of HyKim and as such the following section discusses their features in detail.

\section{$B$ Dynamixels $R X$ modules}

Robotis's Dynamixels were chosen as the servo modules used within HyKim. The RX range of Dynamixels use high quality Swiss Maxon Re-max brushed DC motors. A highly reliable and durable servo motor results when these motors are combined with a full metal gear set and a bearing on the last axis of the output shaft. Table III details the specifications of the Dynamixel RX modules used within HyKim.

Four RX-64 modules were used in areas where large torque was required, these locations being the neck, the hip and the top of each of the rear legs. All Dynamixel modules have a $300^{\circ}$ operating range with a $0.29^{\circ}$ accuracy. The $300^{\circ}$ operating range was essential for the modules located at the top of each of the rear legs, as these must have full range control for quadrupedal and bipedal motions. 
TABLE III

DYNAMIXEL RX MODULE SPECIFICATIONS

\begin{tabular}{|l|l|l|l|l|}
\hline & \multicolumn{3}{|c|}{ Dynamixel Module } \\
\hline & \multicolumn{2}{|c|}{ RX-28 } & \multicolumn{2}{c|}{ RX-64 } \\
\hline Weight (g) & 72 & 125 \\
\hline Dimension (mm) & $35.6 \times 50.6 \times 35.5$ & $40.2 \times 61.1 \times 41.0$ \\
\hline Gear Ratio & $1 / 193$ & $1 / 200$ \\
\hline Voltage & $12 \mathrm{~V}$ & $16 \mathrm{~V}$ & $15 \mathrm{~V}$ & $18 \mathrm{~V}$ \\
\hline Stopping torque (kgf.cm) & 28.3 & 37.7 & 64.4 & 77.2 \\
\hline Speed (sec $\left./ 60^{\circ}\right)$ & 0.167 & 0.126 & 0.188 & 0.157 \\
\hline
\end{tabular}

Dynamixel modules are based around an Atmel ATMega8 microcontroller which allows the modules to be controlled via a multi-drop RS485 network. This allows for operational parameters such as shaft position, operating temperature, load current and input voltage to be returned.

A major advantage gained by using the Dynamixel modules is the distribution of processing. The main control module, HyInt, can simply command the Dynamixel to go to an angular position at a selectable speed. Once the command is issued the main control module can either poll the module to track the shaft position or simply proceed to the next task.

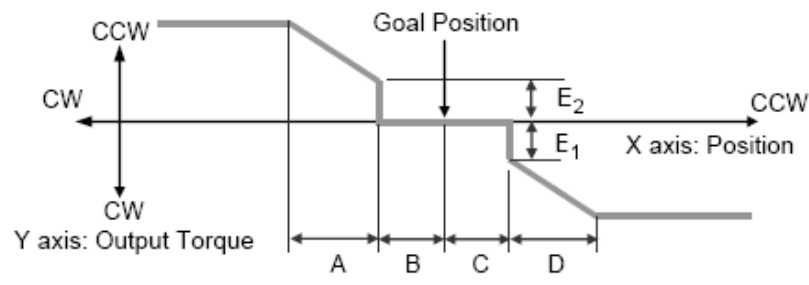

Fig. 8 Compliance characteristic of Dynamixel module [18]

Compliance is defined by Robotis as the relationship between the Dynamixel modules output torque and the shaft position. Fig 8 shows the 6 parameters that are used to define compliance. Careful selection of these parameters can optimise each modules smooth motion to a goal position as well as the ability for the module to absorb external shocks when holding the goal position.

\section{Sensors}

A commercially available USB2.0 webcam is used for HyKim's vision system. This is a high resolution $640 \times 480$ pixel camera with a wide angle lens of $63^{\circ}$, capable of capturing images at $30 \mathrm{fps}$. The webcam plugs directly into a dedicated USB port on HyInt.

Table IV details the remaining sensors available within HyKim. HySense and HySense Lite both have a 6 axis Inertial Measurement Unit (IMU) consisting of a 3 axis accelerometer and a 3 axis gyroscope. HySense also has a

\begin{tabular}{|c|c|c|}
\hline \multicolumn{3}{|c|}{$\begin{array}{c}\text { TABLE IV } \\
\text { HYKIM SENSORS }\end{array}$} \\
\hline & $\begin{array}{l}\text { Body } \\
\text { HySense }\end{array}$ & $\begin{array}{c}\text { Head } \\
\text { HySense Lite }\end{array}$ \\
\hline 6-axis IMU & Yes & Yes \\
\hline Digital Compass & Yes & $\mathrm{n} / \mathrm{a}$ \\
\hline IR Distance Sensor (4 to $30 \mathrm{~cm})$ & Yes (2 of) & \\
\hline IR Distance Sensor $(10$ to $80 \mathrm{~cm})$ & & Yes \\
\hline Capacitive Touch Sensors (3) & not fitted & Yes \\
\hline
\end{tabular}

Digital Compass which can be tilt-compensated using the appropriate accelerometer readings.

HySense and HySense Lite both have the ability to accept 2 IR distance sensors. HySense uses dual 4 to $30 \mathrm{~cm}$ IR distance sensing modules to detect ground based objects, e.g. edge of table, whilst HySense Lite uses a single 10 to $80 \mathrm{~cm}$ IR distance sensing module to assist with image based localisation. Both HySense and HySense Lite have the ability to connect to a capacitive touch sensor board but this is currently only fitted to HySense Lite. Fig. 9 shows HySense Lite and sensors mounted within HyKim's head.

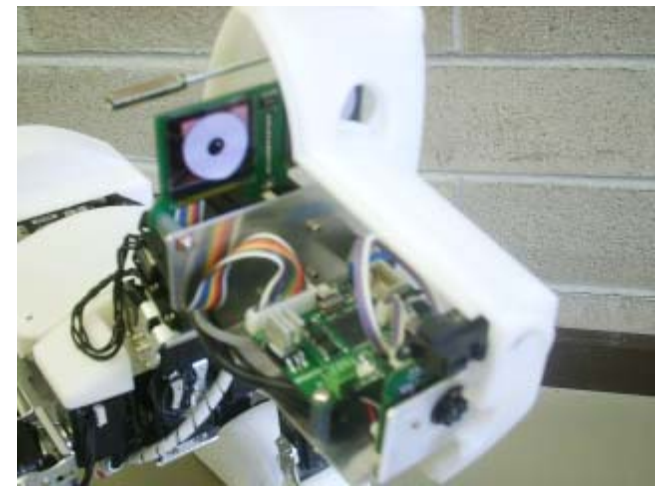

Fig. 9 HySense Lite and sensors mounted in HyKim's head.

$D$ Displays

Fig. 9 shows two $128 \times 128$ pixel OLED's which are located within HyKim's head. These OLED's are connected to and controlled by HySense Lite. These OLED's can serve two purposes,

1) they can be used to allow HyKim to display emotion by graphically displaying eyes in various shapes and colours, and

2) as a diagnostic screen where text and data may be displayed to assist in debugging or monitoring autonomous behaviour.

\section{E Software}

Compulab provide the following operating system images for the CM-iGLX,

\section{1) Linux (Gentoo, 2006)}

2) Win CE (5 only), and

3) $\operatorname{Win} X P$.

An operating system image based on debian etch (Debian 4.0r2) has also been created specifically for HyKim. This image is based around Linux Kernel 2.6.18 and includes make, gec, g++ and python as well as having an apache web server included. This provides the researcher with a stable operating system and a selection of proven development tools.

Functional code for HySense and HySense Lite has been prepared and is pre-installed, but the source code is also readily available should customisation of the code be required. Free development tools, AVR Studio and WinAVR, are made available by Atmel should code customisation be required. Serial boot loaders have been developed for HySense and HySense Lite so that new 
firmware can simply be uploaded to these modules from HyInt over the RS485 network.

\section{FUTURE RESEARCH}

Having created a new and unique robotic platform, our planned research will be briefly presented.

\section{A. Walking Gaits}

Research will be undertaken to establish optimal walking gaits for HyKim as both a quadruped and a biped. Research will also be undertaken to further optimise control of the Dynamixel modules whilst performing walking gaits.

\section{B. Head Stability}

HySense Lite's microcontrollers spare UART has been configured such that it too can communicate over an RS485 network. As shown in Fig. 6, this allows Dynamixel modules to be directly connected to and controlled by HySense Lite. As HySense Lite has an on board 6 axis IMU it is planned to develop a controller for head stability and implement this in HySense Lite's microcontroller. Increasing the head stability whilst moving will reduce the camera 'bounce' and thus reduce imaging processing overheads.

\section{Software}

Plans are being made for a dedicated programming environment and simulator for HyKim.

\section{Rescue}

HyKim is currently being used as the robot in a project investigating ad-hoc wireless MESH networks for Rescue Robots. In this project HyKim will be the rescue robot traversing rough terrain. HyKim's position within the disaster zone will be determined by using the ad-hoc wireless MESH network and triangulation methods. This will involve the addition of pressure sensors on HyKim's feet as well as heat and $\mathrm{CO}_{2}$ sensors mounted in HyKim's head.

\section{CONCLUSION}

Biomimetic principles have been used in the creation of HyKim, a 21 DOF robot bear. Bear anatomy has been researched and bear motions have been observed. This research revealed that two DOF in the back-bone would result in realistic bear movements. A DOF was inserted at the hip with another DOF added to the shoulders.

Compared to a dog, the bulk of a bear gave great flexibility in the selection of servo motors and electronic modules. Robotis's RX range of Dynamixels were selected as the basis for the robot bear. A basic structural skeleton was then created using these modules and where possible readily available Aluminum brackets were used.

A highly distributed processing system was then created. A $500 \mathrm{MHz}$ AMD Geode computer module was selected as the primary processing source; this was incorporated into a modular processing system, HyInt. Two data acquisition modules were then designed, HySense and
HySense Lite. These modules were located in HyKim's body and head respectively. An array of sensors was integrated into HyKim through the data acquisition modules, the main sensors being a 6 axis IMU, in the body and the head, a digital compass and IR distance sensors.

The resulting robot bear is unique and highly functional. HyKim has the ability to mimic bear movements and bear motions, both quadrupedal and bipedal. An open architecture design philosophy was adopted from the outset of this project. The result is a powerful, highly modular robot that researchers can use with the knowledge that all required software, source code and schematics are available.

\section{ACKNOWLEDGMENT}

The author would like to express his sincere thanks to Paul Baker for his help in the development of the prototypes. Thanks are also due to the technical staff in the Discipline of Electrical and Computer Engineering and the team leaders of the NUBot's (2006-2007), without their assistance the creation of HyKim would not have been possible.

\section{REFERENCES}

[1] Sony company, URL: http://www.sony.net

[2] RoboCup organization, URL: http:/www.robocup.org

[3] Standard Platform League (SPL) organization, URL: http://www.tzi.de/spl/bin/view/Website/WebHome

[4] NUBot's organization, URL: http://robots.newcastle.edu.au/

[5] Chalup, S.K., Dickinson, M., Middleton, R.H., Quinlan, M.J., and Turner, P. "Development of a new standard platform for the four legged league of robocup", Multi-Robot Systems: Perception, Behaviours, Learning, and Action, Dagstuhl Seminar Nº6251, Jun. 2006.

[6] Chalup, S.K., Dickinson, M., Fisher, R., Middleton, R.H., Quinlan, M.J., and Turner, P. "Proposal of a kit-style robot as the new standard platform for the four-legged league", Australasian Conference on Robotics and Automation, Auckland, Dec 2006.

[7] Turner, P., "HyKim User Manual" [online], Newcastle, Australia. Tribotix, Jun 2006 [cited Aug. 2, 2008], available from World Wide Web: <http://www.tribotix.info/wiki/index.php/HyKim_v3>

[8] Guynn, D., Trimm, W., Pool, T., "Advanced Black Bear Anatomy and Shot Placement Guide", Montana, USA. Publication No. 8447, IBEP Hunter Responsibility Series, National Bowhunter Education Foundation.

[9] The American Bear Association, "Anatomy of the Black Bear" [online], Minnesota, USA. [cited Oct. 26, 2008], available from World Wide Web: <http://www.americanbear.org/anatomy.htm>

[10] Keck, S., "Bear Anatomy and Physiology" [online], Texas, USA. [cited Oct. 26, 2008], available from World Wide Web: $<$ http://www.bowhunting.net/bearhunting.net/bear2.html $>$

[11] Colla, P., "Bear Anatomy Photos" [online], California, USA. [cited Oct. 26, 2008], available from World Wide Web: $<$ http://www.oceanlight.com/>

[12] Low, A. (Writer), Wilson-Smith, R., Macfarlane, D. (Producers), Brault, S. (Director), 2001 "Bears" [Motion picture]. Montreal, Canada: Primesco.

[13] Dickinson, M., Turner, P., "Development of a biomimetic robotic bear: or is a bare bear bearable ?", IEEE International Conference on Robotics and Biomimetics, Bangkok, Dec 2008.

[14] Aldebaran company, URL: http://www.aldebaran-robotics.com/eng

[15] DasaRobot company, URL: http://genibo.dasarobot.com/english

[16] Robotis company, URL: http://www.robotis.com/eng

[17] Hanson Robotics company, URL: http://hansonrobotics.com

[18] Robotis Staff, "Dynamixel RX-28 User Manual”, v1.10, Robotis, 2006, pp. 26-27. 